\title{
An accurate ray-based offset-to-angle transform from normal moveout uncorrected multicomponent data in a transversely isotropic medium with vertical symmetry axis
}

\author{
Pradip Kumar Mukhopadhyay ${ }^{1}$ and Subhashis Mallick ${ }^{1}$
}

\begin{abstract}
A new ray-based approach for converting multicomponent normal moveout uncorrected offset domain prestack seismic data into angles is presented. From any two-way zero-offset time sample and a given angle of incidence, the exact ray and a few neighboring rays that contribute to the given angle of incidence are traced through the medium to their corresponding source and receiver locations to compute their raypath lengths, traveltimes, and offsets. A polynomial then is fitted locally between these computed offsets and their corresponding traveltimes. Next the traveltimes for the actual offsets on data that lie within these traced rays are computed from this local polynomial fit, and their corresponding time samples are partially stacked and moved to their two-way zero-offset
\end{abstract}

times. Repetition of this procedure for all zero-offset time samples and for all desired angles produces an angle gather. The procedure allows computation of both primary (P-P) and mode-converted (P-SV) angle gathers. Also, by calculating the exact raypath length, an accurate geometric spreading compensation can be computed and applied to these gathers. By using the methodology for a horizontally stratified transversely isotopic medium with a vertical symmetry axis (a VTI medium), it is possible to extract a much more accurate angle-domain response from the ray-based method than is possible from a traditional NMO-based method. Accuracy out to large angles for both primary and mode-converted reflection suggests that this ray-based transform potentially could be used in a multicomponent prestack waveform inversion scheme.

\section{INTRODUCTION}

The variations of primary and converted wave (P-P and $\mathrm{P}-\mathrm{SV})$ reflection amplitudes as functions of the angle of incidence can be calibrated to subsurface lithology and reservoir fluids and are the fundamental basis for amplitude-variationwith-offset-and-angle (AVO/AVA) analysis (Castagna, 1993). For isotropic elastic media, the angular variation of reflection amplitudes depends strongly on the P-wave-to-S-wave velocityratio $\left(V_{\mathrm{P}} / V_{\mathrm{S}}\right)$ contrasts between the reflecting interfaces (Ostrander, 1984). Reservoir parameters, such as P- and S-wave reflectivities, Poisson reflectivity, density reflectivity, and the like, can be estimated from the AVO analysis using a set of weighted stacks (Stolt and Weglein, 1985; Smith and Gidlow, 1987) or from an AVO inversion (Kelly et al., 2001; Skidmore et al., 2001). Alternatively, synthetic data computed in the angle/ray-parameter domain can be compared with the observed prestack seismic data in a waveform inversion scheme to obtain direct estimates of subsurface reservoir properties (Sen and Stoffa, 1991, 1992; Mallick, 1995, 1999; Sen and Roy, 2003). In all these applications, an accurate offset-to-angle transform plays one of the most vital roles.

A current approach to an offset-to-angle transform is based primarily on the work by Todd and Backus (1985) and Resnick (1993), among others. In this procedure, the angle $\theta$ for any twoway zero-offset time sample $t_{0}$ is computed from the relation

$$
\sin \theta=\left(\frac{V_{\text {int }}}{V_{\text {smooth }}}\right)\left\{\frac{x}{\sqrt{x^{2}+\left(V_{\text {smooth }} t_{0}\right)^{2}}}\right\} .
$$

In equation 1, $x$ is the source-to-receiver offset, $V_{\text {smooth }}$ is a spatially varying velocity function derived by smoothing the stacking velocities over a cable length, and $V_{\text {int }}$ is the interval velocity obtained from $V_{\text {smooth. Note that }} V_{\text {int }}$ and $V_{\text {smooth }}$ both are functions of $t_{0}$. Input prestack seismic data first are compensated for spherical divergence loss and corrected for normal moveout

\footnotetext{
Manuscript received by the Editor 29 October 2009; revised manuscript received 22 December 2010; published online 2 May 2011.

${ }^{1}$ University of Wyoming, Department of Geology and Geophysics, Laramie, Wyoming, U. S. A. E-mail: pmukhopa@uwyo.edu; smallick@uwyo.edu; mallick_sub@yahoo.com.

(C) 2011 Society of Exploration Geophysicists. All rights reserved.
} 
(Todd and Backus, 1985). Using equation 1, we then compute the source-to-receiver offset $x$ that corresponds to a given angle $\theta$ and a given zero-offset time sample $t_{0}=n \Delta t$, where $\Delta t$ is the time-sampling interval and $n$ is the sample number.

For band-limited seismic data, the contribution to a given angle does not, however, come from this single offset but instead comes from a range of offsets that lie within a quarter wavelength of the dominant seismic frequency. This concept of the range of offsets contributing to the response to a single angle is similar to the concept of a Fresnel zone, but for an offset-to-angle transform it commonly is called an angle mute pattern or simply an angle mute (Resnick, 1993). In this paper, therefore, we will use the term "angle mute pattern" or "angle mute" to describe this zone.

The seismic trace values for the time sample and for the range of offsets lying within the angle-mute pattern therefore are partially stacked to obtain the response for a given angle and zerooffset time sample (Resnick, 1993). Repeating this operation for all zero-offset time samples and for all desired angles then produces an angle gather. Although such an offset-to-angle transform is for horizontally stratified layers, it can be extended to include dips, following the steps given by Resnick et al. (1987).

The above procedures for an offset-to-angle transform for isotropic layers also can be extended to VTI layers by replacing $V_{\text {smooth }}$ with the corresponding VTI stacking velocity (Alkhalifah, 1997). However, it must be noted that for anisotropic media the angle $\theta$ is the group angle, not the phase angle (Mallick, 2008). For P-P reflections, the offset-to-angle transform computed using the above method produces reasonable results for isotropic media. Its accuracy rapidly diminishes with increasing angles, especially in the presence of anisotropy (Mallick, 2008). Furthermore, for P-S reflections, the NMO equations are complex (Thomsen, 1999), and an offset-to-angle transform does not produce reasonable results even for small angles. The primary purpose of this investigation is to present a ray-based offset-toangle transform that can extract an accurate angle-domain response for VTI media for both primary (P-P) and mode-converted (P-SV) reflections. In contrast to the current NMO-based methodology, to which we refer in this paper as the conventional approach, input data in our transform are offset-domain

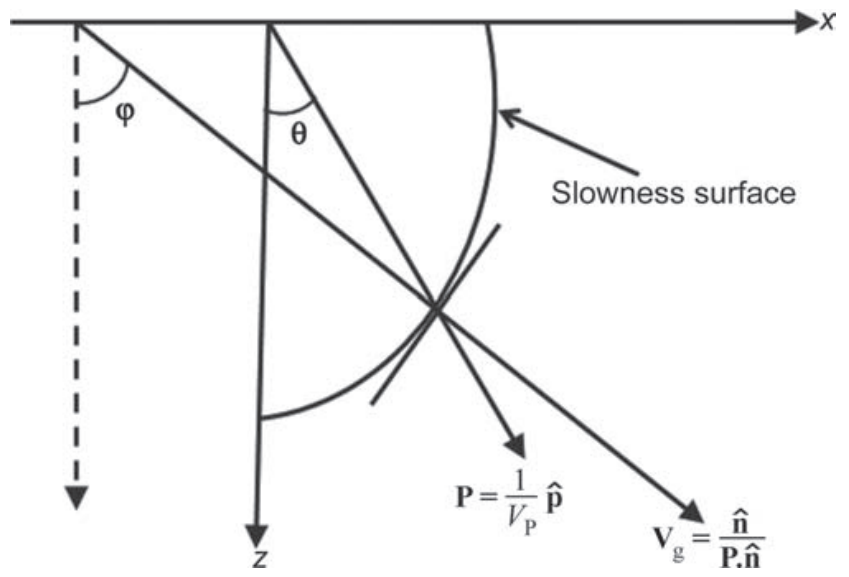

Figure 1. Pictorial representations of phase slowness vector $\mathbf{p}$, group velocity vector $\mathbf{V}_{\mathrm{g}}$, phase angle $\theta$, and group angle $\varphi$ in an anisotropic medium.
NMO-uncorrected prestack seismic data. We ray-trace dynamically with a given angle of incidence from each sample point of $t_{0}$ to the source and receiver locations, thereby giving ourselves the flexibility of obtaining the angle gathers for both P-P and P$\mathrm{SV}$ reflections and displaying them together in zero-offset $\mathrm{P}$ wave times.

Conventional NMO-based angle gathers in the presence of anisotropy are group angle gathers, and for any quantitative interpretation for reservoir properties, they first must be converted to their corresponding phase angles (Mallick, 2008). In a ray-based offset-to-angle transform, we can compute either phase angle gathers or group angle gathers. Lastly, NMO correction is based on a Taylor series approximation of the moveout equation (Yilmaz, 1987). The ray-based offset-to-angle transform, on the other hand, does not make any such approximation. Consequently, we can compute an accurate traveltime, an anisotropic geometric spreading compensation, and an angle mute pattern for the computed gathers.

\section{BACKGROUND THEORY}

For an anisotropic elastic medium, the quasi-P (qP)- and quasi-SV (qSV)-wave velocities vary with the angle (Mallick, 2008). The plane qP- or qSV-wavefields, excited from a point source, propagate outward along the surfaces known as the slowness surfaces, a 2D cross section of which is shown in Figure 1 . Any directed line from the source to the slowness surface represents a plane wave associated with the slowness vector $\mathbf{p}$ and the phase angle $\theta$. The phase velocity $\mathbf{V}_{\mathrm{ph}}$ is also directed along the phase angle $\theta$ and has a magnitude equal to the reciprocal of the slowness vector, that is,

$$
\mathbf{V}_{\mathrm{ph}}=\frac{1}{|\mathbf{p}|} \hat{\mathbf{p}} .
$$

In equation $2, \hat{\mathbf{p}}$ is the unit vector along the slowness direction and $|\mathbf{p}|$ is the magnitude of the slowness vector. The phase velocity corresponding to different phase angles also forms a surface known as the phase-velocity or inverse-slowness surface. Whereas the wavefields of constant phase with a given phase angle $\theta$ propagate outward along the slowness surface, the energy propagates along the direction that is normal to the slowness surface at any given point (see Figure 1). In a lossless elastic medium, the group velocity is directed along this normal with a magnitude equal to the reciprocal of the component of the slowness vector (Auld, 1973); that is,

$$
\mathbf{V}_{\mathrm{g}}=\frac{\hat{\mathbf{n}}}{\hat{\mathbf{n}} \cdot \mathbf{p}},
$$

where $\hat{\mathbf{n}}$ is the unit vector along the direction of the normal to the slowness surface. Note from Figure 1 that for any given slowness vector and the corresponding phase-velocity vector with phase angle $\theta$, there is a corresponding group velocity vector associated with a group angle $\varphi$. In a homogeneous isotropic elastic medium, the propagation velocities of $\mathrm{P}$ - and $\mathrm{SV}$-waves are independent of the propagation direction. Consequently, the slowness surfaces are spheres, the group and phase directions are identical, and the distinction between the phase velocity, group velocity, phase angle, and group angle is not necessary. However, this is different for anisotropic media. 
Assume a VTI medium in which six independent components of the stress tensor $\tau$ are related linearly to the six independent components of the strain tensor e as (Auld, 1973; Thomsen, 1986)

$$
\left(\begin{array}{c}
\tau_{x x} \\
\tau_{y y} \\
\tau_{z z} \\
\tau_{y z} \\
\tau_{z x} \\
\tau_{x y}
\end{array}\right)=\left(\begin{array}{cccccc}
C_{11} & C_{11}-2 C_{66} & C_{13} & 0 & 0 & 0 \\
C_{11}-2 C_{66} & C_{11} & 0 & 0 & 0 & 0 \\
C_{13} & C_{13} & C_{33} & 0 & 0 & 0 \\
0 & 0 & 0 & C_{44} & 0 & 0 \\
0 & 0 & 0 & 0 & C_{44} & 0 \\
0 & 0 & 0 & 0 & 0 & C_{66}
\end{array}\right)\left(\begin{array}{c}
e_{x x} \\
e_{y y} \\
e_{z z} \\
2 e_{y z} \\
2 e_{z x} \\
2 e_{x y}
\end{array}\right) .
$$

Five independent elastic moduli for a VTI medium are, therefore, $C_{11}, C_{13}, C_{33}, C_{44}$, and $C_{66}$. The phase velocity for a VTI medium can be obtained from the solution of the Christoffel equation (Auld, 1973) and has been given by Thomsen (1986). Because a VTI medium is azimuthally isotropic, it is adequate to consider propagation in a vertical $(x-z)$ plane with a given phase angle $\theta$. The quasi-P (qP)- and quasi-SV (qSV)-wave phase velocities $V_{\mathrm{P}}(\theta)$ and $V_{\mathrm{SV}}(\theta)$ then can be expressed as (for details, see Thomsen, 1986)

$$
\begin{aligned}
& V_{\mathrm{P}}^{2}(\theta)=\frac{1}{2 \rho}\left[C_{11} \sin ^{2} \theta+C_{33} \cos ^{2} \theta+C_{44}+D(\theta)\right], \\
& V_{\mathrm{SV}}^{2}(\theta)=\frac{1}{2 \rho}\left[C_{11} \sin ^{2} \theta+C_{33} \cos ^{2} \theta+C_{44}-D(\theta)\right],
\end{aligned}
$$

with

$$
\begin{aligned}
D(\theta)= & \sqrt{\left[\left(C_{11}-C_{44}\right) \sin ^{2} \theta+\left(C_{44}-C_{33}\right) \cos ^{2} \theta\right]^{2}} \\
& +\left(C_{13}+C_{44}\right) \sin ^{2} 2 \theta .
\end{aligned}
$$

The parameter $\rho$ in equations 5 is the density. Now, introducing Thomsen parameters (Thomsen, 1986), we have

$$
\begin{aligned}
& \alpha_{0}=\sqrt{\frac{C_{33}}{\rho}}, \quad \beta_{0}=\sqrt{\frac{C_{44}}{\rho}}, \quad \varepsilon=\frac{C_{11}-C_{33}}{2 C_{33}}, \\
& \gamma=\frac{C_{66}-C_{44}}{2 C_{44}}, \quad \delta=\frac{\left(C_{13}+C_{44}\right)^{2}-\left(C_{33}-C_{44}\right)^{2}}{2 C_{33}\left(C_{33}-C_{44}\right)},
\end{aligned}
$$

and we obtain from equations 5 :

$$
V_{\mathrm{P}}^{2}(\theta)=\alpha_{0}^{2}\left[1+\varepsilon \sin ^{2} \theta+D^{*}(\theta)\right],
$$

and

$$
V_{\mathrm{SV}}^{2}(\theta)=\beta_{0}^{2}\left[1+\frac{\alpha_{0}^{2}}{\beta_{0}^{2}} \varepsilon \sin ^{2} \theta-\frac{\alpha_{0}^{2}}{\beta_{0}^{2}} D^{*}(\theta)\right]
$$

with

$$
D^{*}(\theta)=\frac{1}{2} \varsigma\left\{\left[1+\frac{4 \delta^{*}}{\varsigma^{2}} \sin ^{2} \theta \cos ^{2} \theta+\frac{4(\varsigma+\varepsilon) \varepsilon}{\varsigma^{2}} \sin ^{4} \theta\right]^{\frac{1}{2}}-1\right\} \text {, }
$$

$$
\delta^{*}=\varsigma(2 \delta-\varepsilon)
$$

and

$$
\varsigma=1-\frac{\beta_{0}^{2}}{\alpha_{0}^{2}}
$$

The parameters $\alpha_{0}$ and $\beta_{0}$ in equations 7 are the vertical P-wave velocity and the SV-wave velocity, respectively. Note that the phase velocities given by equations 8 and 9 are exact phase velocities in Thomsen notation, without any weak anisotropic approximation.

Recall from Figure 1 that the group velocity vector is normal to the slowness surface and is given by equation 3 . For a given phase angle $\theta$ with slowness vector $\mathbf{p}$ (Figure 1), the tangent vector $\mathbf{t}$ to the slowness surface is given as (Kreyszig, 1983)

$$
\mathbf{t}=\frac{\mathrm{d} \mathbf{p}}{\mathrm{d} \theta}
$$

From Figure 1, the slowness vector $\mathbf{p}$ can be written as

$$
\mathbf{p}=|\mathbf{p}|(\hat{\mathbf{x}} \sin \theta+\hat{\mathbf{z}} \cos \theta),
$$

where $|\mathbf{p}|$ is the magnitude of the slowness vector, and $\hat{\mathbf{x}}$ and $\hat{\mathbf{z}}$ are the unit vectors along the $x$ - and $z$-axes, respectively. It follows from equations 13 and 14 that

$$
\mathbf{t}=\hat{\mathbf{x}}\left(|\mathbf{p}| \cos \theta+\sin \theta \frac{\mathrm{d}|\mathbf{p}|}{\mathrm{d} \theta}\right)-\hat{\mathbf{z}}\left(|\mathbf{p}| \sin \theta-\cos \theta \frac{\mathrm{d}|\mathbf{p}|}{\mathrm{d} \theta}\right) .
$$

By using equation 14, we also can write the tangent vector $\mathbf{t}$ and the unit normal vector $\hat{\mathbf{n}}$ in terms of phase velocity as

$$
\begin{aligned}
\mathbf{t}= & \frac{1}{\left|\mathbf{V}_{\mathrm{ph}}(\theta)\right|^{2}}\left\{\left[\left|\mathbf{V}_{\mathrm{ph}}(\theta)\right| \cos \theta-\sin \theta \frac{\mathrm{d}\left|\mathbf{V}_{\mathrm{ph}}(\theta)\right|}{\mathrm{d} \theta}\right] \hat{\mathbf{x}}\right. \\
& \left.-\left[\left|\mathbf{V}_{\mathrm{ph}}(\theta)\right| \sin \theta+\cos \theta \frac{\mathrm{d}\left|\mathbf{V}_{\mathrm{ph}}(\theta)\right|}{\mathrm{d} \theta}\right] \hat{\mathbf{z}}\right\},
\end{aligned}
$$

and

$$
\begin{aligned}
\hat{\mathbf{n}}= & \frac{1}{\sqrt{\left|\mathbf{V}_{\mathrm{ph}}(\theta)\right|^{2}+\left(\frac{\mathrm{d}\left|\mathbf{V}_{\mathrm{ph}}(\theta)\right|}{\mathrm{d} \theta}\right)^{2}}} \\
& \times\left\{\left[\left|\mathbf{V}_{\mathrm{ph}}(\theta)\right| \sin \theta+\cos \theta \frac{\left.\mathrm{d}\left|\mathbf{V}_{\mathrm{ph}}(\theta)\right|\right]}{\mathrm{d} \theta}\right] \hat{\mathbf{x}}\right. \\
& \left.+\left[\left|\mathbf{V}_{\mathrm{ph}}(\theta)\right| \cos \theta-\sin \theta \frac{\mathrm{d}\left|\mathbf{V}_{\mathrm{ph}}(\theta)\right|}{\mathrm{d} \theta}\right] \hat{\mathbf{z}}\right\} .
\end{aligned}
$$

In equations 16 and $17, \mathbf{V}_{\mathrm{ph}}$ is the phase velocity of either a $\mathrm{qP}$ or a $\mathrm{qSV}$-wave. If for $\mathbf{V}_{\mathrm{ph}}$ we use the expression for $\mathrm{qP}$-wave phase velocity $V_{\mathrm{P}}$ from equation 8 and the expression for qSVwave phase velocity $V_{\mathrm{SV}}$ from equation 9 , and we plug those terms into equations 16 and 17, respectively, we will provide tangent and normal directions for the respective wavefields. Finally, from the definition of the group velocity vector given by equation 3 , we obtain, after some algebra,

$$
\begin{aligned}
\mathbf{V}_{\mathrm{g}}(\theta)= & \left\{\left[\left|\mathbf{V}_{\mathrm{ph}}(\theta)\right| \sin \theta+\cos \theta \frac{\mathrm{d}\left|\mathbf{V}_{\mathrm{ph}}(\theta)\right|}{\mathrm{d} \theta}\right] \hat{\mathbf{x}}\right. \\
& \left.+\left[\left|\mathbf{V}_{\mathrm{ph}}(\theta)\right| \cos \theta-\sin \theta \frac{\mathrm{d}\left|\mathbf{V}_{\mathrm{ph}}(\theta)\right|}{\mathrm{d} \theta}\right] \hat{\mathbf{z}}\right\} .
\end{aligned}
$$

From equation 18 it follows that the magnitude of the group velocity vector is given as

$$
\left|\mathbf{V}_{\mathrm{g}}(\theta)\right|=\sqrt{\left|\mathbf{V}_{\mathrm{ph}}(\theta)\right|^{2}+\left(\frac{\mathrm{d}\left|\mathbf{V}_{\mathrm{ph}}(\theta)\right|}{\mathrm{d} \theta}\right)^{2}},
$$


and the group angle $\varphi$ is given as

$$
\tan [\varphi(\theta)]=\frac{\tan \theta+\frac{1}{\left|\mathbf{V}_{\mathrm{ph}}(\theta)\right|} \frac{\mathrm{d}\left|\mathbf{V}_{\mathrm{ph}}(\theta)\right|}{\mathrm{d} \theta}}{1-\frac{\tan \theta}{\left|\mathbf{V}_{\mathrm{ph}}(\theta)\right|} \frac{\mathrm{d}\left|\mathbf{V}_{\mathrm{ph}}(\theta)\right|}{\mathrm{d} \theta}} .
$$

Now, for a VTI medium, $\left[\mathrm{d} D^{*}(\theta)\right] /(\mathrm{d} \theta)$ from equation 10 can be derived as

$$
\frac{\mathrm{d} D^{*}(\theta)}{\mathrm{d} \theta}=\frac{\sin 2 \theta}{2 D^{*}(\theta)+\varsigma}\left\{\delta^{*}+2\left[(\varsigma+\varepsilon) \varepsilon-\delta^{*}\right] \sin ^{2} \theta\right\} .
$$

Using equation 21 , we obtain from equation 8

$$
\begin{aligned}
\frac{\mathrm{d}\left|\mathbf{V}_{\mathrm{p}}(\theta)\right|}{\mathrm{d} \theta}= & \frac{1}{2} \frac{\alpha_{0}^{2} \sin 2 \theta}{\left|\mathbf{V}_{\mathrm{p}}(\theta)\right|} \\
& \times\left\{\varepsilon+\frac{1}{2 D^{*}(\theta)+\varsigma}\left[\delta^{*}+2\left(\varsigma \varepsilon+\varepsilon^{2}-\delta^{*}\right) \sin ^{2} \theta\right]\right\},
\end{aligned}
$$

and from equation 9 we have

$$
\begin{aligned}
\frac{\mathrm{d}\left|\mathbf{V}_{\mathrm{SV}}(\theta)\right|}{\mathrm{d} \theta}= & \frac{1}{2} \frac{\alpha_{0}^{2} \sin 2 \theta}{\left|\mathbf{V}_{\mathrm{SV}}(\theta)\right|} \\
& \times\left\{\varepsilon-\frac{1}{2 D^{*}(\theta)+\varsigma}\left[\delta^{*}+2\left(\varsigma \varepsilon+\varepsilon^{2}-\delta^{*}\right) \sin ^{2} \theta\right]\right\} .
\end{aligned}
$$

Plugging the respective expressions for $\left[\mathrm{d}\left|V_{\mathrm{P}}(\theta)\right|\right] /(\mathrm{d} \theta)$ and $\left[\mathrm{d}\left|V_{\mathrm{SV}}(\theta)\right|\right] /(\mathrm{d} \theta)$ from equations 22 and 23 into equations 19 and 20, respectively, provides the analytical expressions of the group velocity and group angle for the $\mathrm{qP}$ - and qSV-waves.

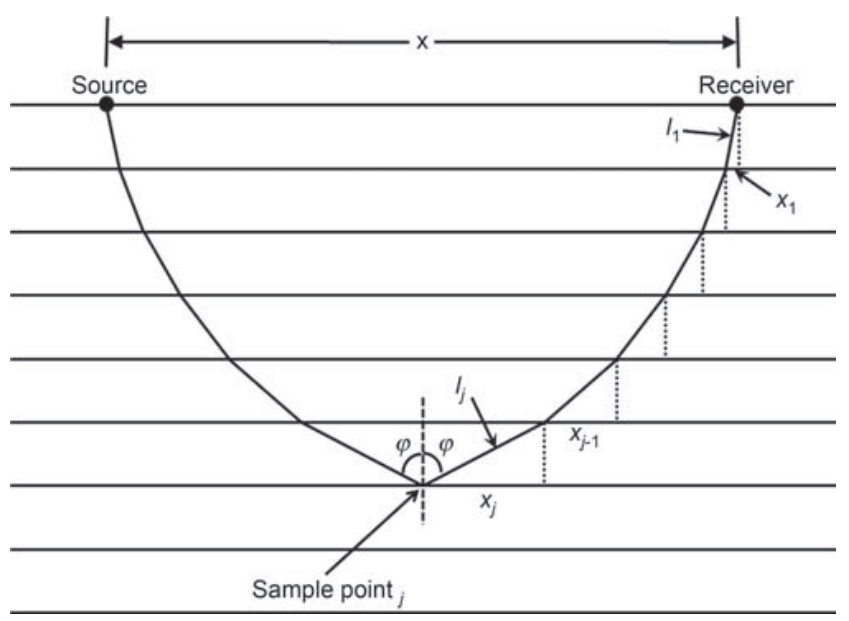

Figure 2. Steps involved in an offset-to-angle transform. For a given sample point and a given angle $\varphi$, we need to ray trace upward to the source and receiver locations to obtain the total offset value $X=\sum_{i=j}^{1} x_{i}$, total raypath length $L=\sum_{i=j}^{1} \ell_{i}$, and total traveltime $T=\sum_{i=j}^{\mathrm{l}} t_{i}$. We need to carry on this process for all time samples and angles to form angle-domain traces.

\section{RAY-BASED OFFSET-TO-ANGLE TRANSFORM}

First, consider a stack of horizontal layers for which the depth of each layer, the vertical velocities, and the anisotropic parameters are given. Because seismic gathers are expressed in time, we first convert this depth model to two-way P-P vertical time, as shown in Figure 2. From any given zero-offset time sample point $(j)$ and for a given group angle (see Figure 2), we calculate the group velocity immediately above the sample. We also calculate the ray parameter that corresponds to the given value of the angle. We then calculate the raypath length, horizontal offset, and traveltime for the layer above the sample. As the ray hits an interface with different material properties, we then calculate the corresponding phase velocity and angle for the new medium by refracting the ray across the interface. After refraction, we calculate the group velocity and group angle for the new layer and continue this procedure until we reach the source and receiver locations. By summing the individual traveltimes, raypath lengths, and horizontal offsets from each segment, we get the total traveltime, raypath length, and horizontal offset. Therefore, the ray-based offset-to-angle transform for P-P reflections for a horizontal stack of layers is

1) For any given zero-offset time sample point $j$, choose the angle $\theta$ (phase or group). If that angle is the phase angle, compute the phase velocity $V_{\mathrm{p}}$ from equation 8 , group velocity $V_{\mathrm{g}}$ from equation 19, and group angle $\varphi$ from equation 20 . From the phase velocity $V_{\mathrm{p}}$ and the phase angle $\theta$, compute the ray parameter $p$, given as $p=\sin \theta / V_{\mathrm{p}}$. If the angle is the group angle, then compute the corresponding phase angle ray parameter $p$, and the group velocity $V_{\mathrm{g}}$.

2) Compute the offset $x_{j}$ at the $j$ th sample point as $x_{j}=(\Delta t / 2) V_{\mathrm{P}}^{j} \tan \varphi$, where $\Delta t$ is the sampling interval and $V_{\mathrm{P}}^{j}$ is the vertical P-wave velocity at sample point $j$. Also calculate the raypath length $\ell_{j}$ and traveltime $t_{j}$ using the group velocity for this sample point (see Figure 2 for details).

3) Repeat steps 1 and 2 to ray trace upward, and calculate offsets $\left(x_{j-1}, x_{j-2}, \ldots, x_{1}\right)$, raypath lengths $\left(\ell_{j-1}, \ell_{j-2}, \ldots, \ell_{1}\right)$, and traveltimes $\left(t_{j-1}, t_{j-2}, \ldots, t_{1}\right)$ for each interface. To apply Snell's law at each interface, it is necessary first to calculate the phase angle from the ray parameter and then to calculate the phase velocities, the group velocities, and the group angle. Once these parameters are obtained, it is straightforward to compute the offsets, raypath lengths, and traveltimes for each interface.

4) Repeat steps 1 through 3 for all samples and angles to obtain the desired offset-to-angle transform.

5) If the source and receiver are located on the same horizontal plane, then the total offset $X$ is $X=2 \sum_{i=j}^{1} x_{i}$, the total raypath length $L$ is $L=2 \sum_{i=j}^{1} \ell_{i}$, and the total traveltime $T$ is $T=2 \sum_{i=j}^{1} t_{i}$. In the case in which the source and receiver locations are at different depths, we calculate the offset, raypath length, and traveltime from the sample point to the source and receiver locations separately, using steps 1 through 3 , and then we add them together to get the total offset, raypath length, and traveltime.

Note that for a P-SV reflection, we need to ray trace from each sample point to the source location by using P-wave properties and to the receiver location by using SV-wave properties. Also note that at every interface, we ray trace using the group 
velocity and angle, but when we move from one interface to the next, we need to refract using the corresponding phase velocities and phase angles. For a given phase angle, it is straightforward to compute the phase velocity, group velocity, and group angle from equations $8,9,19$, and 20 . For a given group angle, however, the phase angle, phase velocity, and group velocity must be computed iteratively even for a VTI medium. To do this, for a given group angle $\varphi$ we first assume the corresponding phase angle $\theta=\varphi$. Using this initial guess of phase angle, we then compute the corresponding group angle and keep modifying the phase angle using a conjugate gradient procedure (Press et al., 1989) until the computed group angle equals $\varphi$ within an acceptable limit of tolerance. Once the phase angle is obtained, we then can compute the phase and group velocities from equations 8,9 , and 19 .

For dipping layers, the dip angles must be taken into consideration in addition to the ray parameter to correctly refract rays across each interface. In the presence of dipping isotropic layers, the same procedure can be used as long as we take the local dips into consideration and ray trace from the given zero-offset sample point to the source and receiver locations separately. However, for dipping anisotropic layers that include VTI, the procedure is not straightforward. Not only do we need to take the local dips into consideration, but we also need to consider the apparent change in the elastic stiffness matrix by rotating the medium by the dip angles using a Bond transformation matrix (Auld, 1973). Extension of this methodology to dipping anisotropic layers is being investigated currently.

Because we work with NMO-uncorrected gathers we not only need to obtain the set of offsets within the angle mute pattern that contribute to a given angle but also their corresponding traveltimes. For a given value of group angle $\varphi_{n}$, we therefore use the above procedure to calculate the traveltime, horizontal offset, and raypath length. To partially stack the traces in the angle mute pattern, we also calculate horizontal offsets and traveltimes for a few more angles that are close to $\varphi_{n}$ and that lie within the angle mute pattern (e.g., $\varphi_{n-1}$ and $\varphi_{n+1}$ ). As is shown in Figure 3, the raypaths $P B$ and $P C$ are such neighboring raypaths of $P A$. As we ray-trace from a given zero-offset time sample, the computed offset values $O A, O B$, and $O C$ for these angles will not necessarily fall on any given offset sample on the input data. This is also true for the calculated traveltimes $B B_{1}, A A_{1}$, and $C C_{1}$. However, we can use these calculated offsets $(O A, O B$, and $O C)$ and corresponding traveltimes $\left(B B_{1}\right.$, $A A_{1}$, and $C C_{1}$ ) and locally fit a polynomial to find the traveltimes for the range of offsets that are present in input data and that lie within the angle mute pattern.

To be specific, assume that for a given angle $\varphi_{n}$ from a given zero-offset time sample $P$ in Figure 3, we ray trace and compute the traveltimes, and also the offsets for angles $\varphi_{n-1}, \varphi_{n}$, and $\varphi_{n+1}$. Assume too that the calculated traveltimes $B B_{1}, A A_{1}, C C_{1}$ and the corresponding offsets $O B, O A$, and $O C$ are given respectively as $t_{n-1}, t_{n}, t_{n+1}$ and $x_{n-1}, x_{n}$, and $x_{n+1}$. We then assume that the time-distance relation in the neighborhood of $\varphi_{n}$ is expressed as a polynomial of the type $t^{2}=c_{0}+c_{2} x^{2}+c_{4} x^{4}$, and we write

$$
\begin{aligned}
& t_{n-1}^{2}=c_{0}+c_{2} x_{n-1}^{2}+c_{4} x_{n-1}^{4} \\
& t_{n}^{2}=c_{0}+c_{2} x_{n}^{2}+c_{4} x_{n}^{4} \\
& t_{n+1}^{2}=c_{0}+c_{2} x_{n+1}^{2}+c_{4} x_{n+1}^{4} .
\end{aligned}
$$

Because the $t_{i}$ and $x_{i}$ values in equation 24 are known through ray tracing, the equation can be solved easily for the coefficients $c_{0}, c_{2}$, and $c_{4}$. Once these coefficients are known, the traveltimes for all the offsets that are within the angle mute pattern between $\varphi_{n-1}$ and $\varphi_{n+1}$ for the input data can be found, partially stacked, and moved to their corresponding zero-offset times. Repeating this procedure for all zero-offset time samples and for all desired values of angles gives us the angle gather. Note that our local polynomials are in general different for different angles and therefore will handle angle-dependent velocity variations correctly. Also note that to demonstrate our procedure of computing traveltimes, in Figure 3 we have shown only one side of the ray tracing from a given sample point to the receiver locations. Clearly, however, to obtain the traveltimes and offsets shown in equation 24 , it is necessary to perform ray tracing from the sample point to the source location also.

A crucial step in our methodology is the accuracy of the polynomial fitting that is used to get the exact time samples. The procedure described above in equation 24 is essentially a three-term polynomial fitting and could be achieved using a Lagrange polynomial (Press et al., 1989). For practical applications, however, we believe that a constrained cubic spline interpolation (Press et al., 1989) is more accurate than a Lagrange polynomial fitting. In the examples shown in this paper, we therefore used a constrained cubic spline interpolation to calculate the traveltimes for the range of offsets described as above. For best results, it is necessary also that the given offset values be spread uniformly within each angle mute. To achieve that, we choose the neighboring angle values while ray tracing in such a way that the calculated offsets fall evenly on either side of the zone. In addition, we also have found that using five closely spaced rays, two on either side of the desired angle, gives the optimum result. An exact calculation of the angle mute pattern is extremely important for the accuracy of our

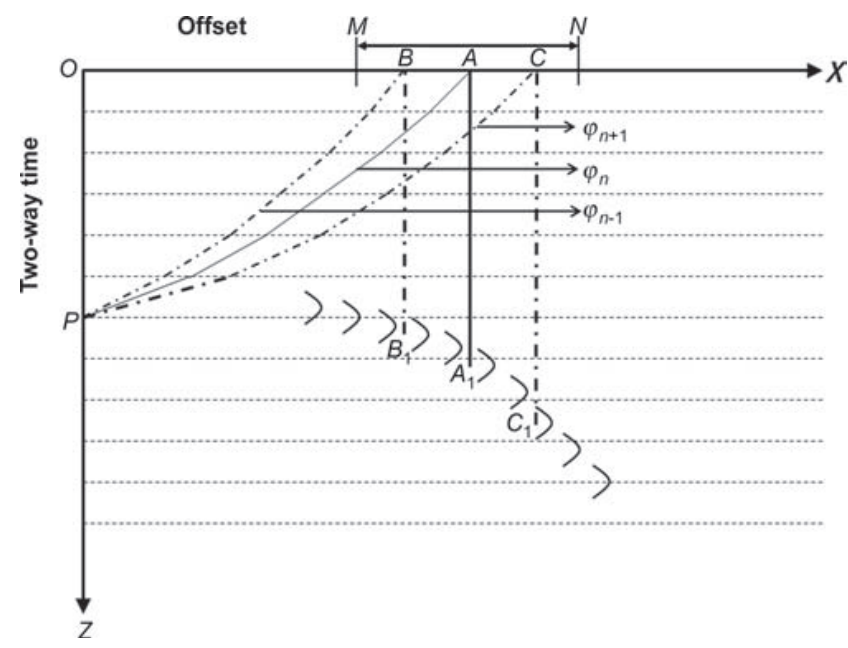

Figure 3. Steps involved in computing an offset-to-angle transform by fitting a polynomial around the given angle $\varphi_{n}$. For a given two-way zero-offset traveltime (point $P$ ) and a given angle $\left(\varphi_{n}\right)$, the corresponding raypath length $(P A)$, traveltime $\left(A A_{1}\right)$, offset $(O A)$, and the angle mute pattern $(M N)$ are shown. For neighboring angle values $\left(\varphi_{n-1}\right.$ and $\left.\varphi_{n+1}\right)$ within this angle mute pattern, the traveltimes and offsets that are required to fit a polynomial locally are also shown. 
method (Monk, 2010). Note that the angle mute pattern is a function of offset, angle, depth, and dominant seismic frequency, and an incorrect calculation of the angle mute pattern can cause destructive interference to the computed angle-domain responses.

\section{EXAMPLES WITH DISCUSSION}

Figure 4 shows a multilayer anisotropic model in two-way Pwave time and depth. This anisotropic model is derived from a real well-log and anisotropic velocity analysis of prestack seismic data. Figure 5 shows the vertical and radial component of the response in offset domain, computed for this model. These responses were computed using a wave-equation-based modeling algorithm for a stratified anisotropic medium (for details, see Mallick and Frazer, 1987, 1988, 1990, 1991).

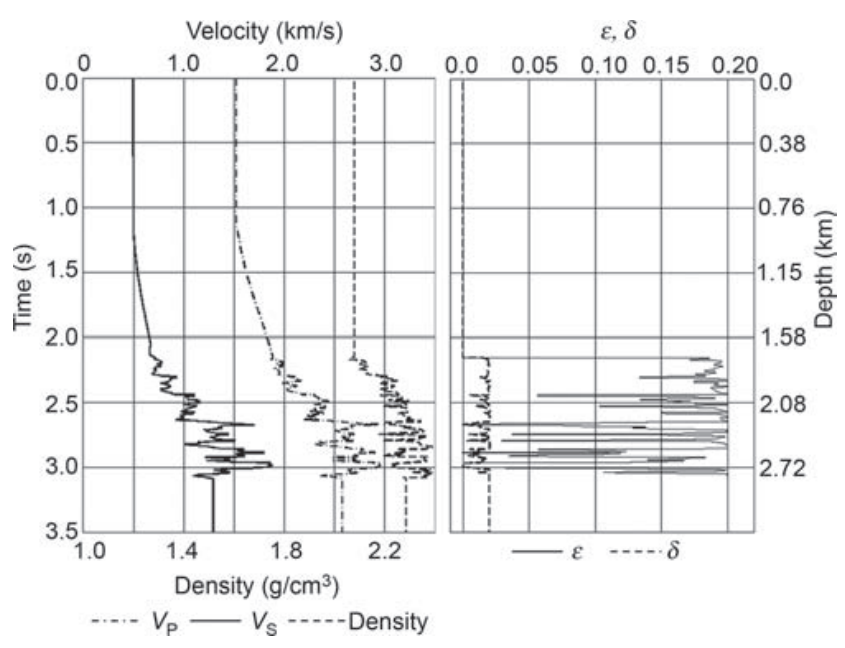

Figure 4. Multilayer anisotropic earth model in P-P time and depth, derived from a real well log.

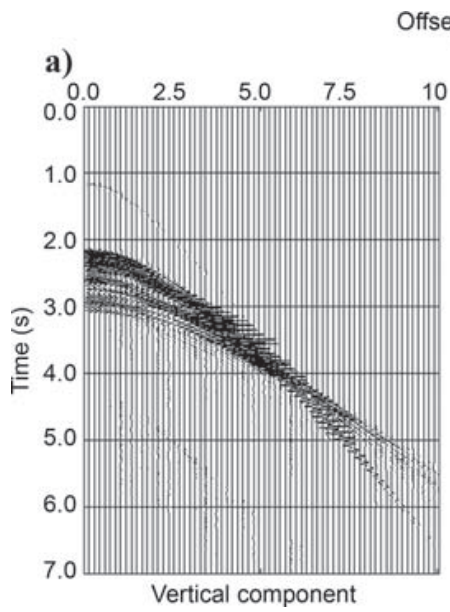

b)

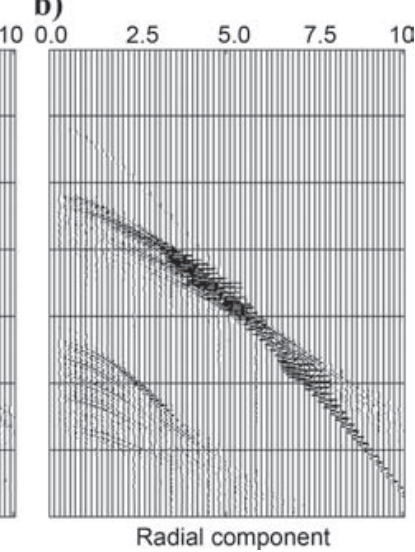

Figure 5. (a) Vertical component and (b) radial component of the synthetic seismic response, computed from the model shown in Figure 4.
Figure 6 shows the P-P angle gathers. Figure $6 \mathrm{a}$ is the angle gather computed from the synthetic offset gather using a conventional method - that is, after NMO correction of the vertical component of the response, computation of the range of offsets for the given angles from equation 1, and partial stacking of the traces over the angle mute pattern, as described by Todd and Backus (1985) and Resnick (1993). Because our input model is VTI, we replaced the isotropic stacking velocities in equation 1 with their corresponding anisotropic stacking velocities (see Alkhalifah, 1997 for details). Figure 6b is the angle gather computed by convolving the source wavelet with an exact (Zoeppritz) P-P reflection series for different angles, and Figure $6 \mathrm{c}$ is the new ray-based angle gather. Note that the conventional and ray-based angle gathers are computed from the same input model shown in Figure 4, and the Zoeppritz gather is shown for reference to common practice.

Figure 7 shows the converted wave angle gathers for the same model. Only the ray-based gather is compared with the Zoeppritz gather in this figure. For clarity, the converted wave angle gathers of Figure 7 were magnified by a factor of ten compared with the P-P gathers of Figure 6. Notice that P-SV angle gathers are shown in two-way P-P time, not in P-SV time.

The example shown in Figures 6 and 7 demonstrates the superiority of the ray-based offset-to-angle transformation compared with the results from a conventional approach. Notice that the P-P events in the conventional gather are bent upward with increasing angles (Figure 6a). Additionally, the reflection amplitudes in the conventional angle gather tend to dim with increasing traveltime. Although such bending of events is controlled by the choice of the VTI parameters $\varepsilon$ and $\delta$, the main reason for such bending and dimming of amplitudes is the increasing inaccuracy of the NMO equation for large offsets. NMO equations for the mode-converted reflections are complex even for an isotropic medium. By accurately computing the mode-converted

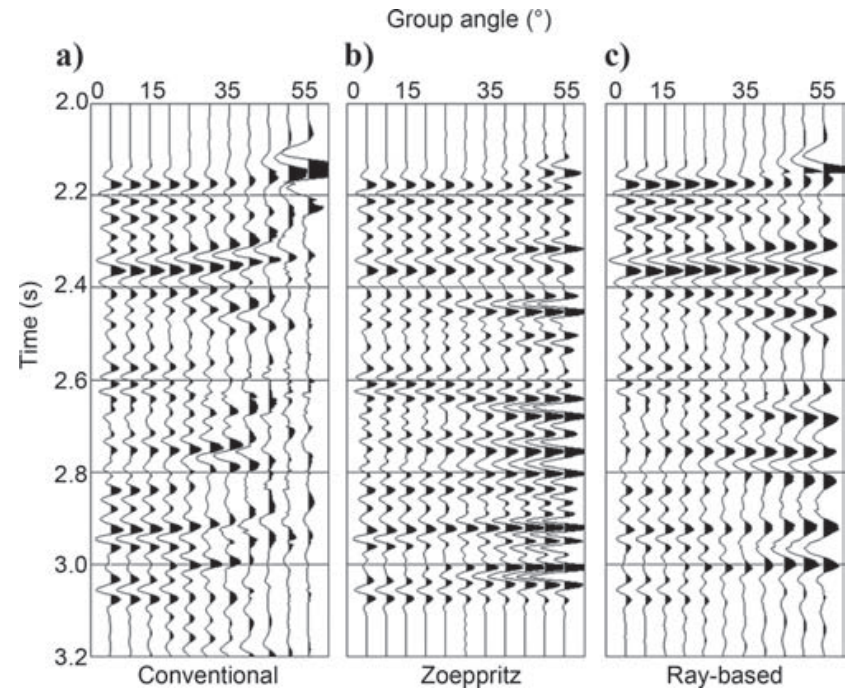

Figure 6. Angle-domain primary (P-P) seismograms computed using the model shown in Figure 4 and from the offset-domain vertical-component response shown in Figure 5a. (a) P-P angle gather computed using a conventional approach, (b) P-P Zoeppritz angle gather, and (c) P-P angle gather using the new ray-based method. 
traveltimes, not only have we computed accurate angle gathers but we also have shown a convenient way of referencing them to P-wave time (Figure 7) so that they can be compared jointly with P-P reflections in an AVO/AVA analysis. By computing the exact traveltimes through ray tracing, we are able to improve the quality of the computed angle gathers drastically (Figures 6c, 7b).

In Figures 6 and 7, notice that even though we used exactly the same wavelet, the Zoeppritz gathers (Figures 6b and 7a) appear to have higher frequency content than the wave equation gathers have (Figures 6a, 6c, 7b), especially with increasing time. The primary reason for this is the fact that the wave equation angle gathers, irrespective of whether they are conventional or ray-based, are obtained from the offset-domain synthetics. They are computed using a wave equation methodology that included all effects of wave propagation, such as interbed multiple reflections and mode conversions. These propagation effects in a finely layered medium cause an apparent loss of frequency, known as the O'Doherty-Anstey effect or the stratigraphic-filtering effect (for details, see Banik et al., 1985a and Banik et al., 1985b). Zoeppritz gathers, on the other hand, are computed by convolving the angle-dependent reflection coefficients with the given wavelet and therefore do not contain any such effect. Consequently, the frequency content is higher in the Zoeppritz gather than in the wave equation angle gathers.

Because of wavefield interference effects, demonstrating the accuracy of the ray-based offset-to-angle transform is difficult

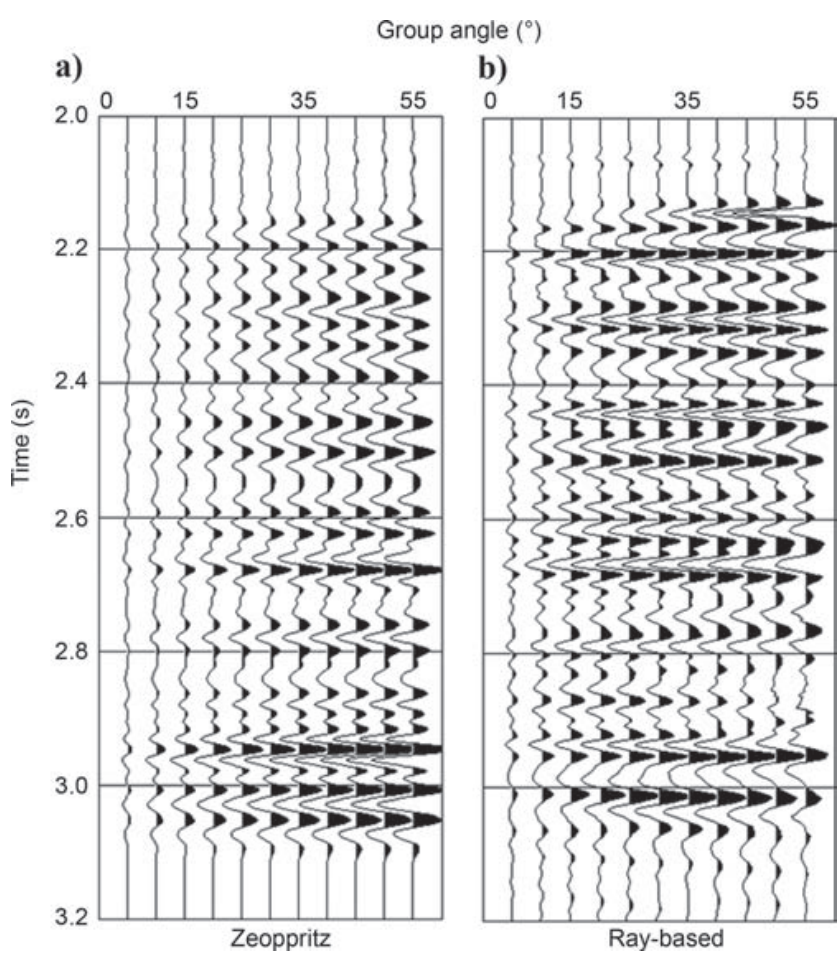

Figure 7. Angle-domain mode-converted (P-SV) gathers computed using the model shown in Figure 4 and from the offset-domain radial-component response shown in Figure 5b. (a) Zoeppritz angle gather and (b) ray-based angle gather. Note that both these angle gathers are displayed in P-wave time. from the gathers shown in Figures 6 and 7. Figure 8 shows a simple five-layer VTI model and Figure 9 shows the computed vertical (9a) and radial component (9b) synthetic seismograms for that model. Figures 10 and 11 show, respectively, P-P and $\mathrm{P}-\mathrm{SV}$ angle gathers extracted from those synthetic responses. Figures 12 and 13 show the extracted amplitudes respectively of all four P-P and P-SV reflection events seen in Figures 10 and 11 .

The five-layer examples shown in Figures 10 and 11 allow direct comparison of the extracted amplitudes with Zoeppritz amplitudes. Note that for P-P reflections, shown in Figure 12, the extracted reflection amplitudes from the conventional angle gather deteriorate with increasing time as a result of the presence of anisotropy in the model. Even though Event 4 (Figure 12d) appears to be correct for the conventional gather, note that this reflection is very weak for large angles, and the apparent proximity of the conventional angle gather reflections to the Zoeppritz reflections for this event results from the fact that the

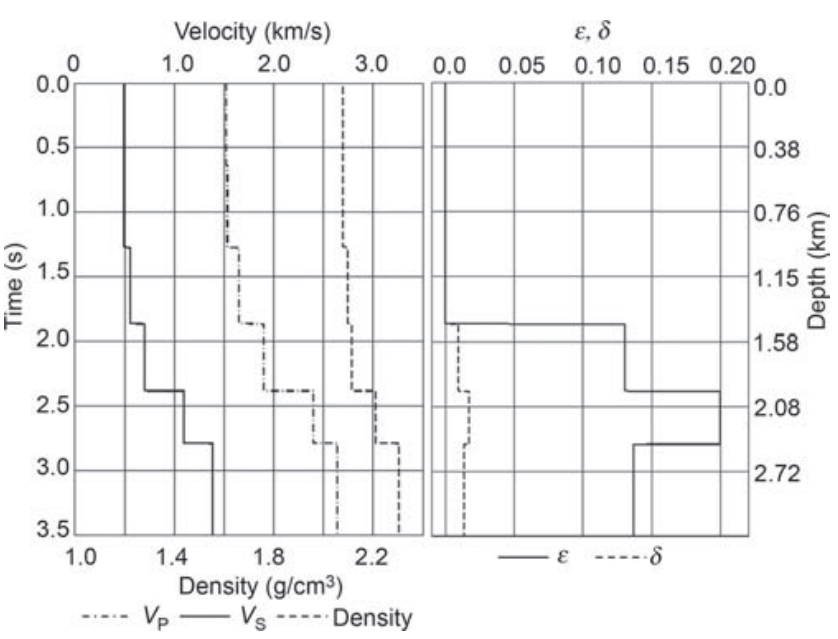

Figure 8. A simple five-layer VTI model in P-P time and depth.

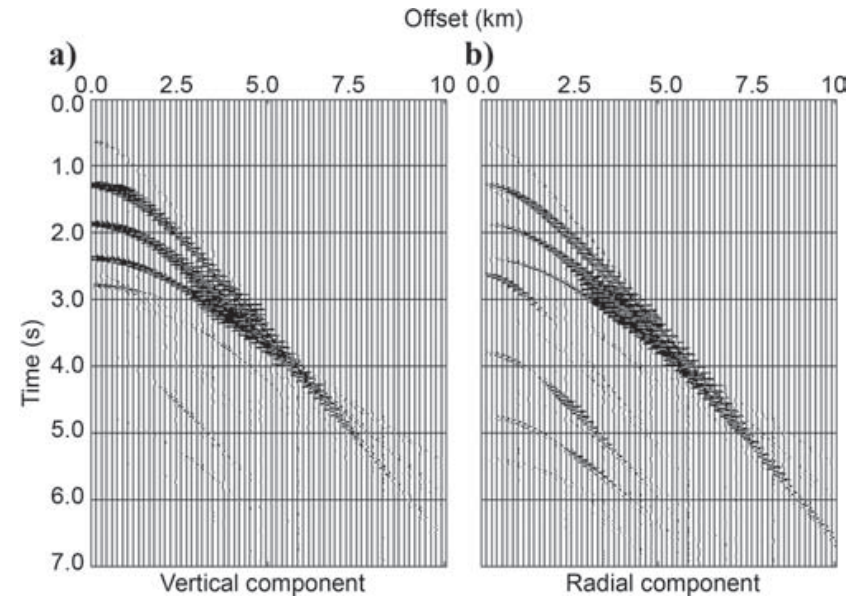

Figure 9. (a) Vertical-component and (b) radial-component synthetic seismic responses for the model shown in Figure 8. 
reflection amplitudes themselves are nearly zero. The ray-based P-P gathers, on the other hand, are very close to the Zoeppritz reflections for all four events shown in Figure 12.

For mode-converted reflections (Figure 13), notice that the ray-based amplitudes match very closely with Zoeppritz reflec- a)

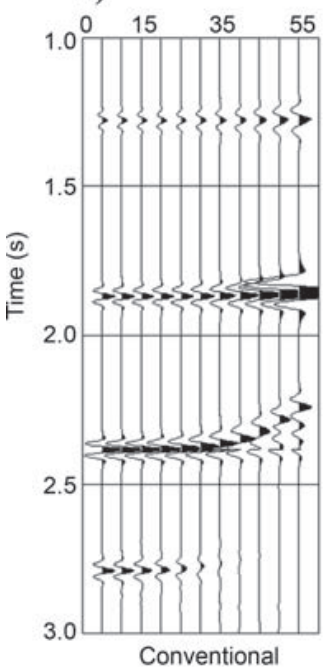

b)

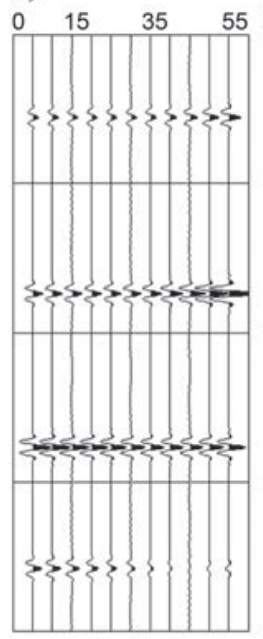

Zoeppritz c)

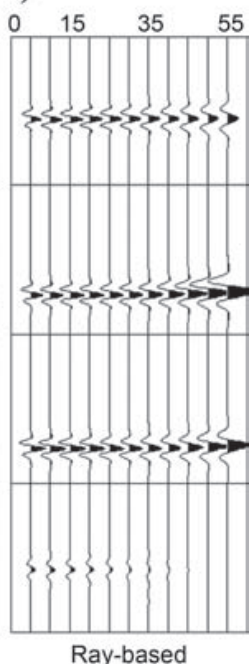

Figure 10. Angle-domain primary (P-P) seismograms computed using the model shown in Figure 8 and the vertical component response shown in Figure 9a. (a) P-P angle gather computed using a conventional approach, (b) P-P Zoeppritz angle gather, and (c) $\mathrm{P}-\mathrm{P}$ angle gather using the ray-based method.

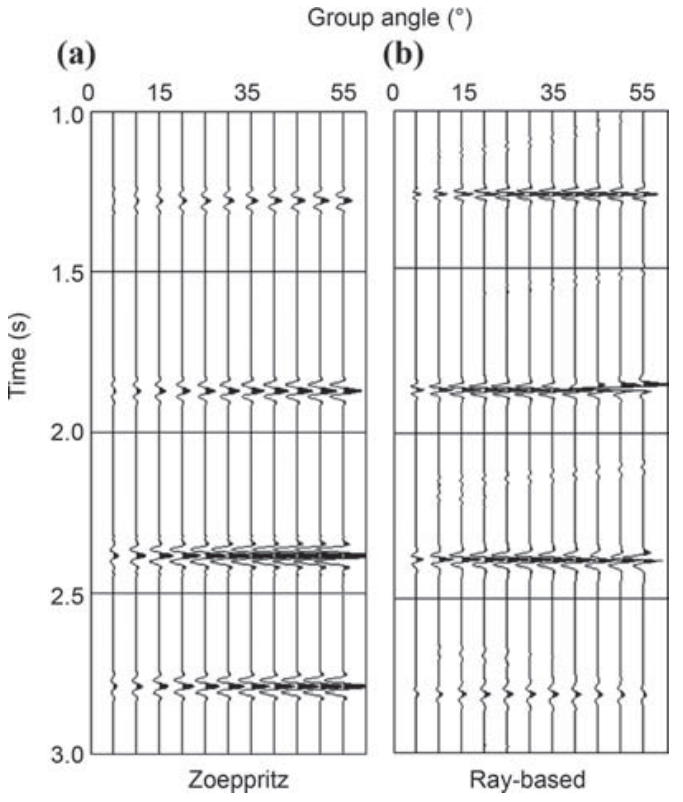

Figure 11. Angle-domain mode-converted (P-SV) gather computed using the model shown in Figure 8 and the radial-component response shown in Figure 9b. (a) Zoeppritz angle gather and (b) ray-based angle gather.

tions for events 1 and 4 . For events 2 and 3, however, the match is good only to $50^{\circ}$. That is because in the offset gather (Figure $9 \mathrm{~b}$ ), the radial component of response at large offsets is affected by interference from the multiply reflected $\mathrm{P}$-wave modes for these reflections. This interference is apparent in the P-SV angle gather for these events beyond $50^{\circ}$.

Figures 14 and 15 show the sensitivity of the ray-based offset-to-angle transform to the input anisotropic parameters. From the five-layer anisotropic model of Figure 8, we generated two additional models: (1) an isotropic model created by setting $\varepsilon=\delta=0$ for the entire model, and (2) a $10 \%$ anisotropic model created by setting $\varepsilon=0.10$ for maximum anisotropy in the model of Figure 8 and leaving $\delta$ values as they are. Figures 14 and 15 compare the conventional and ray-based angle gathers

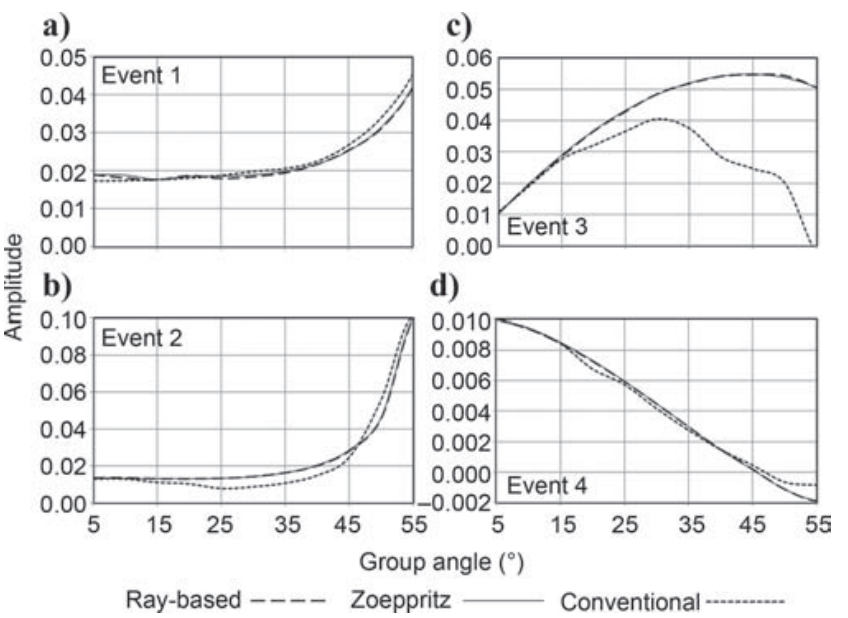

Figure 12. Comparisons of P-P reflection amplitudes for all four reflection events of Figure 10. Amplitudes for (a) event 1, (b) event 2 , (c) event 3 , and (d) event 4.

a)

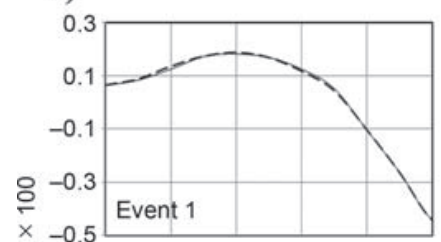

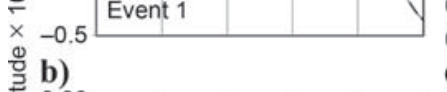

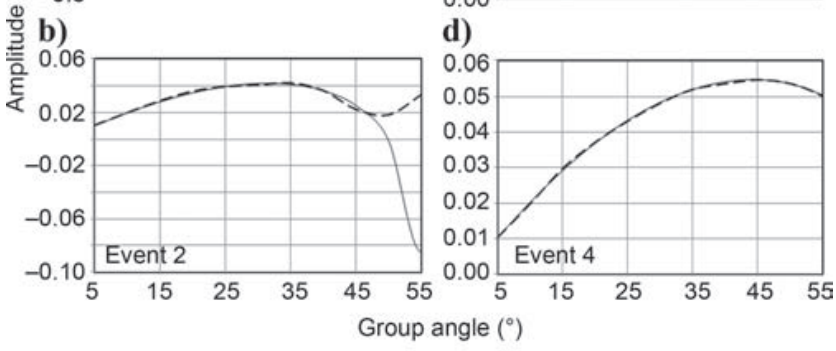

Ray-based ---- Zoeppritz

Figure 13. Comparisons of P-SV reflection amplitudes for all four reflection events of Figure 11. Amplitudes for (a) event 1, (b) event 2, (c) event 3, and (d) event 4. 
for these two new models and the original model of Figure 8. In subsequent discussion, we refer to these three models as (1) the isotropic model, (2) the $10 \%$ anisotropic model, and (3) the $20 \%$ anisotropic model. Figure $14 \mathrm{a}$ is the conventional anglegather for the isotropic model, Figure $14 \mathrm{~b}$ is the conventional angle-gather for the $10 \%$ anisotropic model, and Figure $14 \mathrm{c}$ is the conventional angle-gather for the $20 \%$ anisotropic model. In Figure $15 \mathrm{a}, \mathrm{b}$, and $\mathrm{c}$ we have the respective ray-based angle gathers for the same set of models.

The conventional NMO-based offset-to-angle transform provides reasonable results for isotropic media, but its accuracy in anisotropic media diminishes rapidly with increasing angle and with increasing anisotropy. From Figure 14, note that the conventional angle gathers are increasingly inaccurate as anisotropy increases in the model. Ray-based angle gathers (Figure 15), on the other hand, are not. The accuracy of ray-based angle gathers out to large angles both for primary (P-P) and for mode-converted (P-SV) reflections is the result of the accuracy of the transform in computing the large-angle traveltimes. This accuracy out to large angles in fact places a strong constraint on model estimation and potentially be used in a multicomponent waveform inversion. The use of ray-based angle gathers for such an inversion currently is being investigated and will be discussed in a separate paper.

Finally, note that the ray-based transform can properly account for the difference between the phase angles and the group angles. It is important to be aware of the differences between the phase and group velocities and angles when one is dealing with anisotropic AVO analyses; otherwise, any quantitative interpretation for reservoir properties will lead to erroneous results. Figure 16 is a plot of group angle versus phase angle and reflection coefficients for two sets of models. Both models consist of a top shale layer and a bottom sand layer. Elastic constants for these layers were taken from the real well data of Figure 4 and are given in Table 1 . The group angle versus the

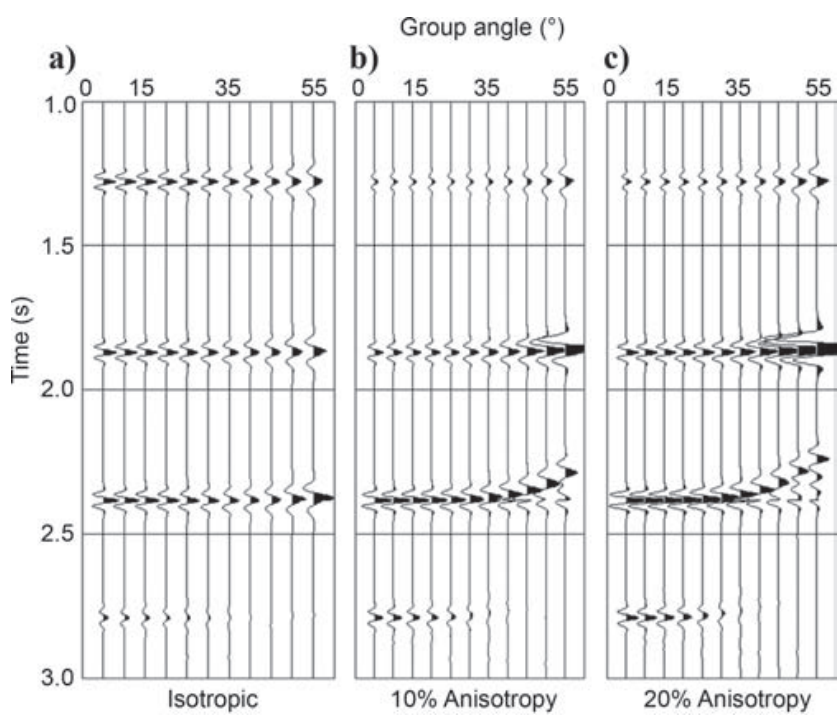

Figure 14. Analysis of the sensitivity of the conventional offsetto-angle transform to the input anisotropic model. Conventional offset-to-angle transform using (a) the isotropic model, (b) the $10 \%$ anisotropic model, and (c) the $20 \%$ anisotropic model. phase angle and the P-P and P-SV reflection coefficients for model 1 are shown respectively in Figure 16a, b, and c. Note that these reflection coefficients are exact anisotropic reflection

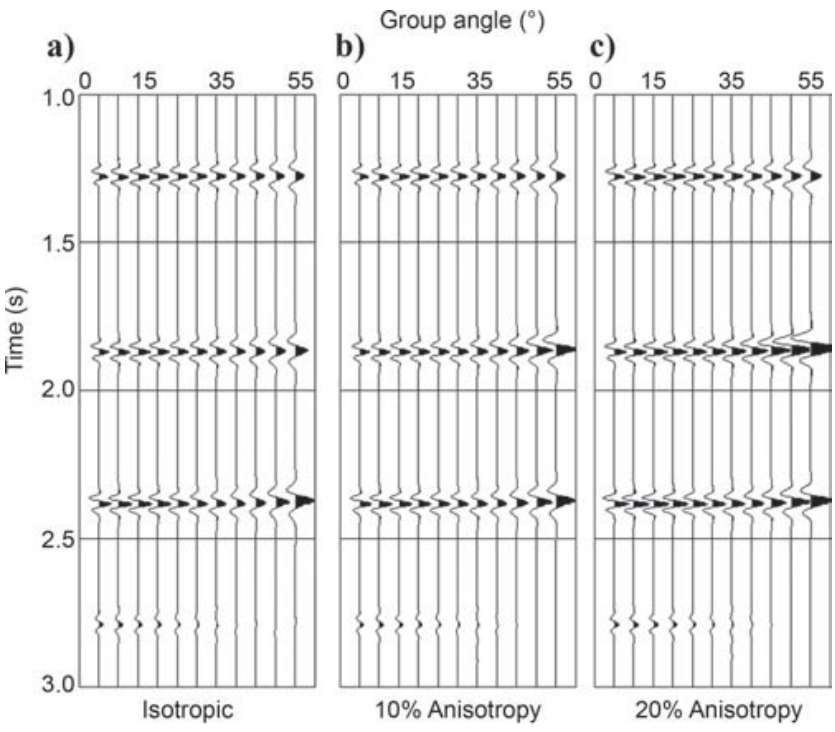

Figure 15. Same sensitivity analysis as Figure 14, but using the ray-based offset-to-angle transform. a)

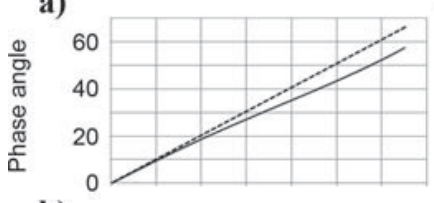

b)

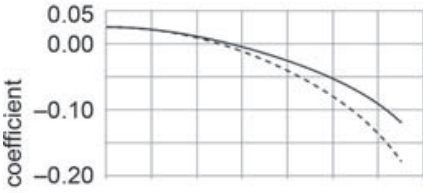

응 c)

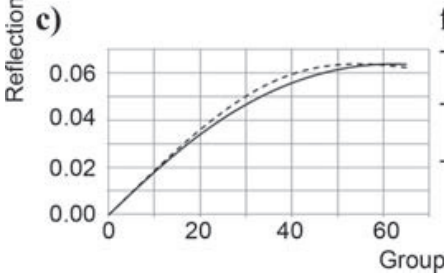

d)

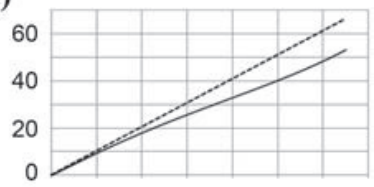

e)

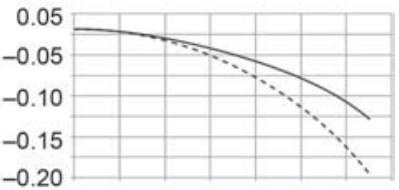

f)

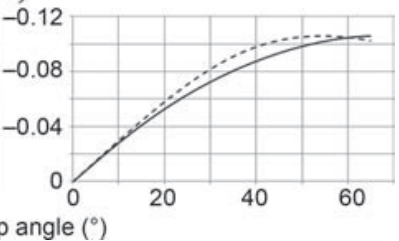

Figure 16. Relation between group angle and phase angle and P-P and P-SV reflection coefficients for two anisotropic models extracted from real well data. Both of the models consist of a top shale layer and a bottom sand layer. The model parameters are given in Table 1. (a) Dependence of phase angle on group angle for model 1. The solid curve is the phase angle as a function of group angle, and the dashed curve is shown as a reference in which the phase angle is assumed to be equal to the group angle. (b) P-P reflection coefficient as a function of group angle for model 1. The solid curve is computed by transforming the group angles to their corresponding phase angles and then computing the reflection coefficients for those phase angles, whereas the dashed curve is computed by assuming that the group angles are equal to the phase angles. (c) The same as (b), but for the P-SV reflection coefficient as a function of group angle for model 1. (d-f) The same as (a-c), but for model 2. 
Table 1. Models used for computing the group angle and phase angle and the P-P and P-SV reflection coefficients for two anisotropic models, shown in Figure 16.

\begin{tabular}{llllll}
\hline & \multicolumn{2}{c}{ Model 1 } & & \multicolumn{2}{c}{ Model 2 } \\
\cline { 2 - 3 } \cline { 5 - 6 } $\begin{array}{l}\text { Model } \\
\text { parameters }\end{array}$ & $\begin{array}{c}\text { Top } \\
\text { layer }\end{array}$ & $\begin{array}{c}\text { Bottom } \\
\text { layer }\end{array}$ & & $\begin{array}{l}\text { Top } \\
\text { layer }\end{array}$ & $\begin{array}{c}\text { Bottom } \\
\text { layer }\end{array}$ \\
\hline$V_{\mathrm{P} 0}(\mathrm{~km} / \mathrm{s})$ & 2.581 & 2.372 & & 2.170 & 2.295 \\
$V_{\mathrm{S} 0}(\mathrm{~km} / \mathrm{s})$ & 1.269 & 1.474 & & 0.888 & 1.209 \\
$\rho\left(\mathrm{gm} / \mathrm{cm}^{3}\right)$ & 2.2 & 2.19 & & 2.26 & 2.2 \\
$\varepsilon$ & 0.12 & 0.002 & & 0.2 & 0.002 \\
$\delta$ & 0.01 & 0.0002 & 0.02 & 0.0002 \\
\hline
\end{tabular}

coefficients computed using the procedure outlined by Mallick and Frazer (1991). Because group angles are the ones that determine the traveltimes of seismic reflections, we chose to use the group angle as the independent variable and to show the phase angle and reflection coefficients as functions of the group angle.

Although the anisotropy for the first model is relatively weak (12\%), significant differences still exist between the group angles and phase angles for this model, as seen in Figure 16a. Whereas the solid curve in Figure 16a shows the group-angle versus phase-angle relation for model 1 , for convenience we also have included in this figure a dashed line along which the phase angle is equal to the group angle. Notice that when the group angle is $60^{\circ}$, the corresponding phase angle is approximately $52^{\circ}$ for model 1 . In the reflection-coefficient curves of Figure $16 \mathrm{~b}$ and $\mathrm{c}$, the curves with solid lines are the reflection coefficients computed by first converting the group angles to their corresponding phase angles and then computing the reflection coefficients for those phase angles. The dashed curves, on the other hand, were computed by assuming that the group angles are equal to the phase angles. The computed results for the second model are shown in Figure 16d, e, and f. Notice that the anisotropy in the top shale layer in the second model is $20 \%$ and significant differences exist between the phase angles and the group angles. In real seismic data, although our extracted angles are group angles, the reflection amplitudes will be proportional to the reflection coefficients for their corresponding phase angles. In other words, these reflection amplitudes will be proportional to the solid curves shown in Figure 16b, c, e, and f. In AVO/AVA analysis, on the other hand, if we ignore this fact and assume the group angle to be equal to the phase angle, the reflection amplitudes for the same models will follow the dashed curves. Consequently, an AVO/AVA-based inversion of the angle-domain data in which the difference between group angles and phase angles is ignored will result in incorrect reservoir properties.

\section{CONCLUSIONS}

Computing an accurate offset-to-angle transform is crucial to predicting subsurface lithology and fluid properties from seismic data. Whereas a conventional NMO-based transform is increasingly inaccurate with increasing angles, especially in the presence of anisotropy, a ray-based method allows accurate calcula- tion of angle gathers. It also allows computation of converted wave angle gathers in P-wave times, thereby offering a convenient way to jointly analyze primary and mode-converted reflection responses in AVO/AVA applications. Because the raybased approach accurately models large-angle traveltime, in principle it places a stronger constraint on model estimation. Therefore, the ray-based approach potentially can be used to compute angle gathers effectively from observed seismic data and to match them iteratively with synthetic angle gathers in a multicomponent prestack waveform inversion scheme.

\section{ACKNOWLEDGMENTS}

We thank Phil Anno, the SEG Associate Editor, and three anonymous reviewers, whose constructive comments greatly helped improve the quality of this paper. This research is funded by the U.S. Department of Energy Grant DE-NT0004730 and DE-FE0001160 .

\section{REFERENCES}

Alkhalifah, T., 1997, Velocity analysis using nonhyperbolic moveout in transversely isotropic media: Geophysics, 62, 1839-1854.

Auld, B. A., 1973, Acoustic waves in solids: 1, John Wiley and Sons.

Banik, N., I. Lerche, J. R. Resnick, and R. T. Shuey, 1985a, Stratigraphic filtering, Part II: Model spectra: Geophysics, 50, 2775-2783, doi:10.1190/1.1441898.

Banik, N., I. Lerche, and R. T. Shuey, 1985b, Stratigraphic filtering, Part I: Derivation of the O'Doherty-Anstey formula: Geophysics, 50, 27682774, doi:10.1190/1.1441897.

Castagna, J. P., 1993, AVO analysis - Tutorial and review, in J. P. Castagna and M. M. Backus, eds., Offset-dependent reflectivity: Theory and practice of AVO analysis: SEG Investigations in Geophysics No. 8, 3-36.

Kelly, M., C. Skidmore, and D. Ford, 2001, AVO inversion, Part I: Isolating rock property contrasts: The Leading Edge, 20, 320-323, doi: $10.1190 / 1.1438940$.

Kreyszig, E., 1983, Advanced engineering mathematics: John Wiley and Sons.

Mallick, S., 1995, Model-based inversion of amplitude-variation-with-offset data using a genetic algorithm: Geophysics, 60, 939-954, doi:10.1190/1.1443860.

- 1999, Some practical aspects of prestack waveform inversion using a genetic algorithm: An example from the east Texas Woodbine gas sand: Geophysics, 64, 326-336, doi:10.1190/1.1444538.

_, 2008 , Interpretation of angle gathers for transversely isotropic medium: 78th Annual International Meeting, SEG, Expanded Abstracts, 2937-2941.

Mallick, S., and L. N. Frazer, 1987, Practical aspects of reflectivity modeling: Geophysics, 52, 1355-1364, doi:10.1190/1.1442248.

, 1988, Rapid computation of multioffset vertical seismic profile synthetic seismograms for layered media: Geophysics, 53, 479-491, doi:10.1190/1.1442479.

_ 1990 , Computation of synthetic seismograms for stratified azimuthally anisotropic media: Journal of Geophysical Research, 95, B6, 8513-8526, doi:10.1029/JB095iB06p08513.

- 1991, Reflection/transmission coefficients and azimuthal anisotropy in marine seismic studies: Geophysical Journal International, 105, no. 1, 241-252, doi:10.1111/j.1365-246X.1991.tb03459.x.

Monk, D. J., 2010, Fresnel-zone binning: Fresnel-zone shape with offset and velocity function: Geophysics, 75, no. 1, T9-T14, doi:10.1190/1.3294576.

Ostrander, W. J., 1984, Plane-wave reflection coefficients for gas sands at nonnormal angles of incidence: Geophysics, 49, 1637-1648, doi:10.1190/1.1441571.

Press, W. H., B. P. Flannery, S. A. Teukolsky, and W. T. Veterling, 1989, Numerical recipes: Cambridge University Press.

Resnick, J. R., 1993, Seismic data processing for AVO and AVA analysis, in J. P. Castagna and M. M. Backus, eds., Offset-dependent reflectivity: Theory and practice of AVO analysis: SEG Investigations in Geophysics No. 8, 175-189.

Resnick, J. R., P. Ng, and K. Larner, 1987, Amplitude versus offset analysis in the presence of dip: 57th Annual International Meeting, SEG, Expanded Abstracts, 617-620.

Sen, M. K., and I. G. Roy, 2003, Computation of differential seismograms and iteration adaptive regularization in prestack waveform inversion: Geophysics, 68, 2026-2039, doi:10.1190/1.1635056. 
Sen, M. K., and P. L. Stoffa, 1991, Nonlinear one-dimensional seismic waveform inversion using simulated annealing: Geophysics, 56, 1624 1638, doi:10.1190/1.1442973.

, 1992, Genetic inversion of AVO: The Leading Edge, 11, 27-29, doi:10.1190/1.1436845.

Skidmore, C., M. Kelly, and R. Cotton, 2001, AVO inversion, Part 2: Isolating rock property contrasts: The Leading Edge, 20, 425-428, doi:10.1190/1.1438966.

Smith, G. C., and P. M. Gidlow, 1987, Weighted stacking for rock property estimation and detection of gas: Geophysical Prospecting, 35, 9931014, doi:10.1111/j.1365-2478.1987.tb00856.x.
Stolt, R. H., and A. B. Weglein, 1985, Migration and inversion of seismic data: Geophysics, 50, 2458-2472, doi:10.1190/1.1441877.

Thomsen, L., 1986, Weak elastic anisotropy: Geophysics, 51, 1954-1966, doi:10.1190/1.1442051.

_ 1999, Converted-wave reflection seismology over inhomogeneous, anisotropic media: Geophysics, 64, 678-690, doi:10.1190/1.1444577.

Todd, C. P., and M. M. Backus, 1985, Offset-dependent reflectivity in a structural context: 55th Annual International Meeting, SEG, Expanded Abstracts, 586-588.

Yilmaz, O., 1987, Seismic data processing: SEG Investigations in Geophysics No. 2 